\title{
Characterisation of contact parameters of sand grains to be used for discrete element modelling
}

\author{
K. Balamonica ${ }^{1}$, Siang Huat Goh $^{1}$ \\ ${ }^{1}$ National University of Singapore, Singapore - 119077, Singapore
}

\begin{abstract}
Discrete element method (DEM) is a numerical technique that models the material as a combination of discrete particles which interact with each other through the contacts springs. While modelling soil in finite element method (FEM), approximations are made which considers soil as a continuous material. Hence certain properties such as void ratio which are intrinsic properties of the soil cannot be directly modelled or monitored. FEM also requires the failure criteria to be defined to predict the post elastic behaviour of the soil. DEM overcomes such drawbacks and the particle contacts are the only parameters that has to be defined. In the present work the contact parameters for sand grains are estimated for the purpose of being modelled in DEM, using direct measurement approach. The parameters required for defining the contact springs between the particles are the young's modulus, coefficient of restitution, friction coefficient, Poisson's ratio and density. The estimated parameters from the experiments were used to model the soil behaviour in an oedometer test. The obtained results from the DEM modelling are compared with the experimental results to benchmark the parameters estimated for the sand grains.
\end{abstract}

\section{Introduction}

The discrete element method (DEM) is a numerical approach which models the material as an ensemble of discrete particles which interact with each other in every timestep [1]. This is in contrary to the finite element method (FEM) which assumes the material to be continuous in nature. Soil being an assembly of discrete particles it is more appropriate to model the soil by DEM than by FEM.

The particles in the DEM interact with each other with the help of normal and tangential springs (Fig. 1). The properties of the spring directly influence the macroscopic behaviour of the soil mass. Hence calibrating the contact parameters is a crucial step in modelling a material in discrete element method. The contact parameters can be calibrated by two approach namely bulk calibration approach and the direct measurement approach [2]. In the bulk calibration approach the particle contact parameters are adjusted in trial and error method to mimic the macroscopic behaviour of the soil [3]. Direct measurement approach measures the microscopic properties experimentally and the contact parameters are derived based on the measured values [4,5]. In the present study the soil particles are modelled based on the hertz contact model and the contact parameters are derived by a direct measurement approach.

\section{Hertz Contact model}

Hertzian contact model is a non-linear contact model in which the contact force depends on the penetration depth.
It considers the spherical nature of the contacting bodies and the area of contact is a circle of growing radius. The force displacement relationship is given by [6]:

$$
F=K \delta^{n}
$$

where $\delta$ is the penetration, $\mathrm{K}$ is the stiffness of the material. The factor $n$ is equal to $3 / 2$ and it is derived by considering the contacting area. The complexity of the Hertz theory in calculating the shear contact was overcome by using the Mindlin model for the tangential forces [7]. This combined method was given by [8].

The Hertzian pressure relationship between two spheres of radius $\mathrm{R}_{\mathrm{i}}$, elastic modulus $\mathrm{E}_{\mathrm{i}}$, and Poisson's ration $\nu_{\iota}$ is given by:

$$
P(r)=P_{0}\left(1-\left(\frac{r}{a}\right)^{2}\right)^{1 / 2}
$$

where:

$$
\begin{aligned}
& P_{0}=\left(\frac{3 P}{2 \pi a^{2}}\right) \\
& a=\left(\frac{3 P R *}{4 E *}\right)^{1 / 3}
\end{aligned}
$$

From the pressure relationship the force can be expressed as

$$
\begin{gathered}
F_{n}=\frac{4}{3} \sqrt{R *} U_{n}^{0.5} U_{n} \\
R^{*}=\frac{R_{i} R_{j}}{R_{i}+R_{j}}
\end{gathered}
$$

\footnotetext{
"Corresponding author: balamonica@u.nus.edu, gohsianghuat@,nus.edu.sg
} 


$$
\frac{1}{E^{*}}=\frac{1-\vartheta_{i}^{2}}{E_{i}}+\frac{1-\vartheta_{j}^{2}}{E_{j}}
$$

where $\mathrm{E}^{*}$ is the equivalent Young's modulus and $\mathrm{R}^{*}$ is the equivalent radius of the contacting spheres. The tangential stiffness is found by using the Hertz-Mindlin theory. Tangential stiffness accounts for the slipping that occurs in the contact. The tangential force is given by:

$$
F_{t}=\frac{2}{3}\left(8 G * \sqrt{R *} U_{n}^{0.5}\right) U_{t}
$$

where $G^{*}$ is the equivalent shear modulus, $U_{t}$ is the tangential displacement or overlap. The Hertz-Mindlin model gives better results when the particles are assumed to be of spherical shape [9].

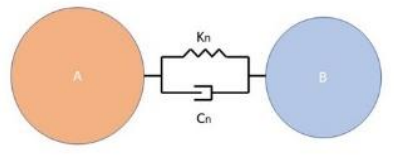

(a) Normal Contact

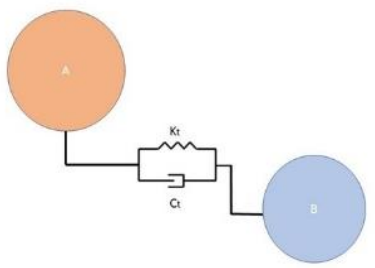

(b) Tangential Contact
Fig. 1. Contact model used in the DEM model

Young's modulus, Poisson's ratio, coefficient of restitution and friction angle are the important parameters that are to be used for a DEM model. In the present study, direct measurement approach is used to characterise the contact parameters. Young's modulus of a microscopic particles such as sand grain is very difficult to measure experimentally. But precise test such as Nano indentation can efficiently determine the microscopic modulus of the materials [10-12]. Friction angle of the soil can be measured through incline plane experiment $[13,14]$ as well as by direct shear test $[15,16]$. A drop test which measures the height of bounce of the particle dropped can be used to determine the coefficient of restitution [17]. In the current paper, though the properties are measured by a direct measurement approach, their ability to predict the macroscopic properties is studied by benchmarking the DEM model created by using the measured properties with respect to the experimental results obtained from physical testing.

\section{Characterization of the contact parameters for filter sand grade 6}

For the present study filter sand grade 5.5 having particle size ranging from $1.5-3 \mathrm{~mm}$ is used. The particles were characterised for the microscopic properties by physical tests. The specific gravity of the sand is found to be 2.63 and the poisons ratio is taken as 0.25 . The list of tests performed to characterize the contact parameters are given in Table 1.
Table 1. Tests conducted to characterise the soil sample

\begin{tabular}{|c|c|}
\hline Soil parameter & Tests conducted \\
\hline Youngs modulus & Nano indentation tests \\
\hline Friction angle & Direct shear test \\
\hline Coefficient of restitution & Drop test \\
\hline
\end{tabular}

\subsection{Nano indentation test}

Hysitron TI Premier Nano-indenter (Bruker) is used in the present study to perform the Nano-indentation test. The soil grains were separated and the samples for the test is prepared by fixing the soil grains on to the microscopic slides using epoxy adhesive. The prepared samples are shown in the Fig. 2.

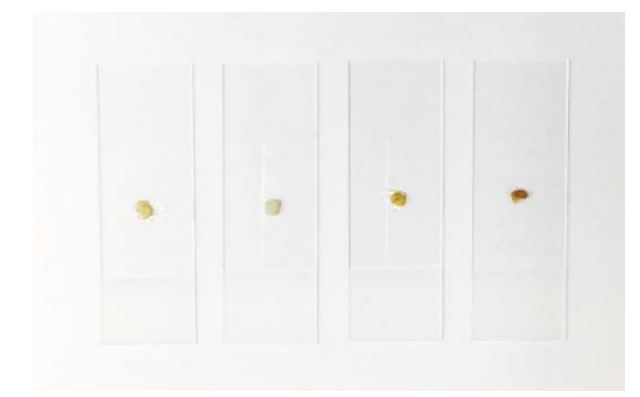

Fig. 2. Samples for the Nao-indentation test

The Nano-indentation tests are performed at room temperature using a Berkovich tip (pyramidal diamond tip with three sides) and triboscan software. Sand samples S_1 to S_6 are loaded and unloaded in a force-controlled approach to a force level of $300 \mu-\mathrm{N}$ whereas samples S_7 and $\mathrm{S} \_$are loaded and unloaded in a displacementcontrolled approach to make an indentation depth of $30 \mathrm{~nm}$ and $50 \mathrm{~nm}$ respectively. The force displacement response of each sand particles is given in the Fig. 3 .

Table 2 Young's modulus of the sand particles found from Nano-indentation tests

$\begin{array}{cc}\text { Sand samples } & \text { E (GPA) } \\ \text { S_1 } & 64.14 \\ \text { S_2 } & 61.38 \\ \text { S_3 } & 63.73 \\ \text { S_4 } & 62.16 \\ \text { S_5 } & 63.08 \\ \text { S_6 } & 60.04 \\ \text { S_7 } & 68.10 \\ \text { S_8 } & 77.20 \\ \text { Average } & \mathbf{6 4 . 9 8}\end{array}$




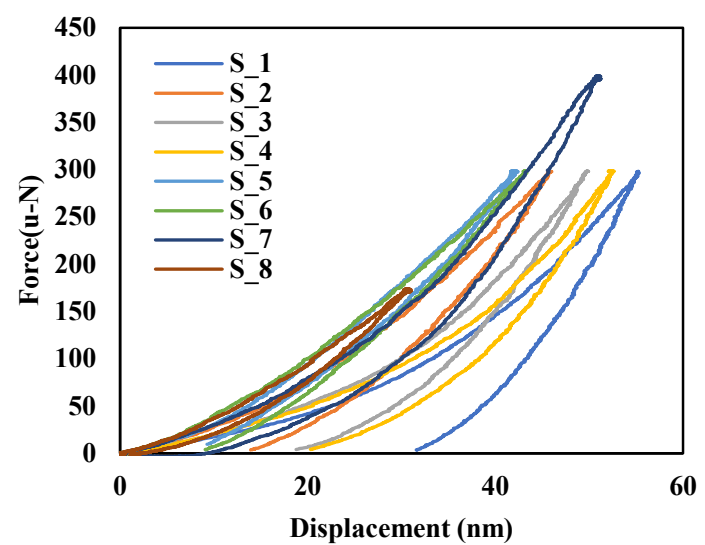

Fig. 3. Force displacement response of the sand particles in a Nano-indentation test

The elastic modulus of the materials is calculated from the unloading curve. The average of the modulus is used for the modelling the sand particles in DEM.

\subsection{Direct shear test}

Interparticle friction angle is an important parameter to be used to model a material in a discrete element method. Direct shear test is used to determine the angle of shearing resistance. Three sets of sand specimens are tested under a normal stress of $40 \mathrm{kpa}, 80 \mathrm{kpa}$ and $160 \mathrm{kpa}$ and the corresponding shear stress is used to arrive at the angle of the shearing resistances.

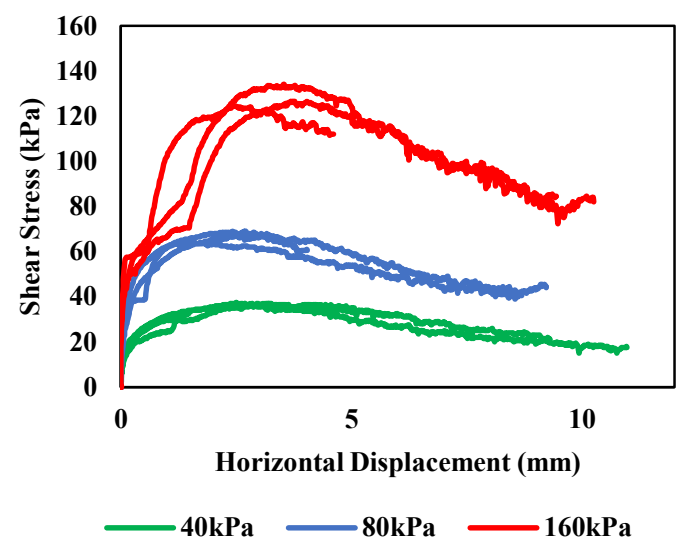

Fig. 4. Variation of shear stress with the horizontal displacement for a sand specimen in direct shear tests

Fig. 4 shows the variation of shear stress with the horizontal displacement. The failure envelope is plotted between the peak shear stress and normal stress and the friction angle is found from the Fig. 5. Though the angle of shearing resistance doesn't directly measure the interparticle friction angle, in the present analysis the inter particle friction angle is taken as $39^{\circ}$.

\subsection{Drop test}

Coefficient of restitution is defined as the ratio of the post impact velocity to the pre-impact velocity. The coefficient of restitution is an important parameter especially in dynamic problems. The coefficient of restitution is given by

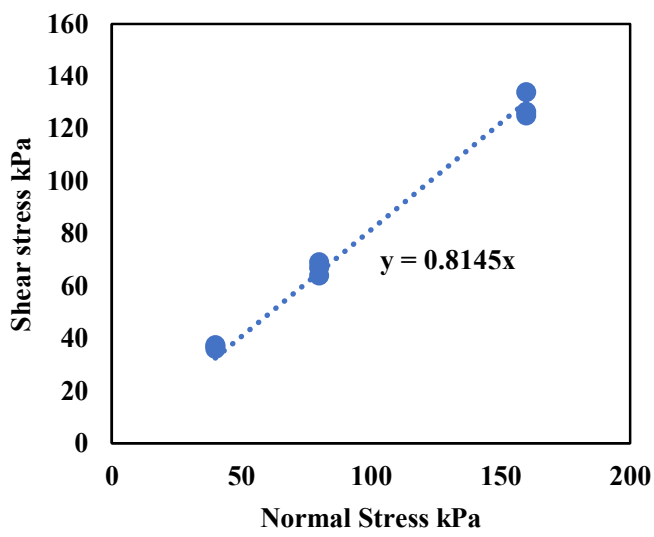

Fig. 5. relationship between the shear stress and normal stress

$$
e=\frac{V_{2}}{V_{1}}=\left(\frac{h_{2}}{h_{1}}\right)^{2}
$$

where $V_{2}$ and $V_{1}$ are the velocities after and before the impact, $h_{2}$ and $h_{1}$ are the rebound height and height of drop respectively.

It can be measured by a simple drop test. In the present work, MotionBLITZ EoSens high speed camera is used at a frame rate of $375 \mathrm{fps}$. A soil particle was dropped from a height on to a smooth surface and its motion is tracked through the camera. The drop height and rebound height are measured from the same datum in pixels and the ratio is used to find the coefficient of restitution (Fig. 6). Since the sand particles are not purely spherical in shape and are of varied sizes the coefficient restitution was calculated randomly and the average of all was taken as the restitution value (Table 3 ).

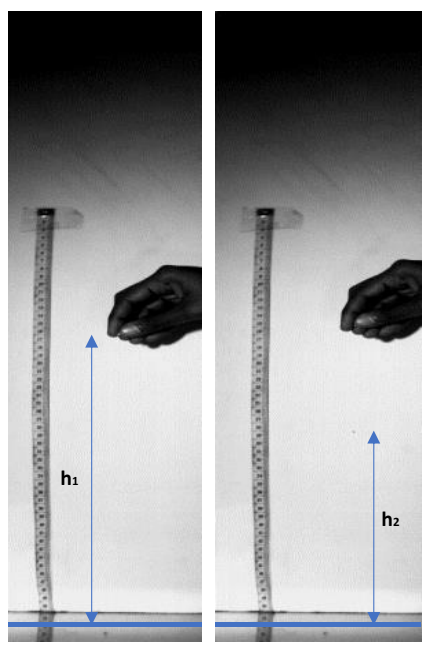

Fig. 6. Drop test to measure the coefficient of restitution 
Table 3. Measurement of coefficient of restitution

\begin{tabular}{cccc}
\hline S.No & $\begin{array}{c}\text { Initial } \\
\text { height } \\
\text { (Pixels) }\end{array}$ & $\begin{array}{c}\text { Bouncing } \\
\text { height } \\
\text { (Pixels) }\end{array}$ & e \\
1 & 387.46 & 157.54 & 0.64 \\
2 & 381.64 & 149.5 & 0.63 \\
3 & 292.24 & 257.56 & 0.94 \\
4 & 315.44 & 278.99 & 0.94 \\
5 & 331.88 & 216.59 & 0.81 \\
\hline
\end{tabular}

Table 4. Contact parameters to be used for discrete element modelling

\begin{tabular}{cc}
\hline Parameter & Value \\
$\begin{array}{c}\text { Coefficient of } \\
\text { restitution }\end{array}$ & 0.79 \\
Coefficient of friction & $0.8145(\Phi=39)$ \\
Youngs modulus & $65 \mathrm{GPA}$ \\
Specific gravity & 2.63 \\
\hline
\end{tabular}

\section{Validation of the contact parameters to be used for discrete element modelling}

Contact characterization is an important aspect of the discrete element modelling as the DEM results are greatly influenced by the input parameters. To validate if the parameters estimated by direct measurements as given in the Table 4 can model the sand behaviour, an oedometer test is performed.

\subsection{One dimensional compression test on sand specimen}

An automatic oedometer apparatus which can apply load pneumatically on to the specimen is used. The mould of $5 \mathrm{~cm}$ diameter and $2 \mathrm{~cm}$ height is filled with soil particles. And the entire test is carried out in dry state. The soil sample is subjected to stress levels varying from $3000 \mathrm{kPa}$ to $4500 \mathrm{kPa}$ as given in the Table 5. The axial displacements measured using a LVDT is plotted against the force measured.

Table 5. Oedometer test details

\begin{tabular}{|c|c|c|}
\hline Sample no & Peak stress (kPa) & Peak load (N) \\
\hline S1 & 3000 & 5888 \\
\hline S2 & 3000 & 5888 \\
\hline S3 & 3500 & 6875 \\
\hline S4 & 4000 & 7850 \\
\hline S5 & 4500 & 8833 \\
\hline
\end{tabular}

\subsection{Discrete element model}

A DEM model is created to closely follow the physical oedometer experiment conducted. LAMMPS (Largescale Atomic/Molecular Massively Parallel Simulator) an open source molecular dynamics software is used for the present study [18].

A cylindrical rigid container of diameter $5 \mathrm{~cm}$ and height $2 \mathrm{~cm}$ identical to the experiment is created. Though the sand is angular in shape the DEM particles are modelling as spherical elements. The particles were created to closely follow the particle size distribution of the sand (Fig. 7). The particles were created in a region above the container and they are allowed to settle under gravity. The number of particles to be created were calculated based on the mass of the sand taken for the experiment. A rigid plate made of particles of $1 \mathrm{~mm}$ diameter is introduced into the model. The plate is also let to settle under gravity and the whole assembly is allowed to attain an equilibrium state. Fig. 8 shows the final sample prepared in the DEM. The particles in the DEM interact with each other by the contact springs and dampers in the normal as well as tangential directions. The properties of the spring are derived based on the contact parameters estimated by direct measurement (Table 4).

One dimensional compression test on the DEM model was performed in a force-controlled approach. The specimen is loaded to generate a stress of $3600 \mathrm{kPa}$ by applying equivalent force on to each particles of the plate. The displacement of the sample is relatively measured with respect to the movement of the plate.

The force displacement relationship obtained from the DEM modelling is in good agreement with the range of results obtained from the oedometer tests (Fig. 9).

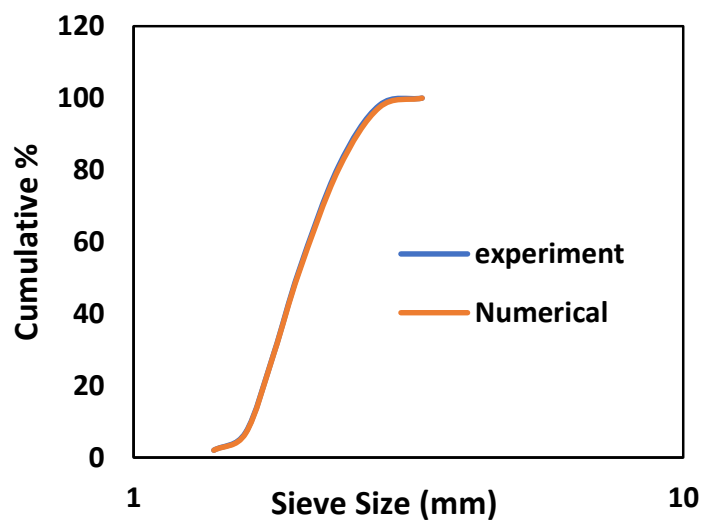

Fig. 7. Particle size distribution between the experimental sand sample and the LAMMPS model 


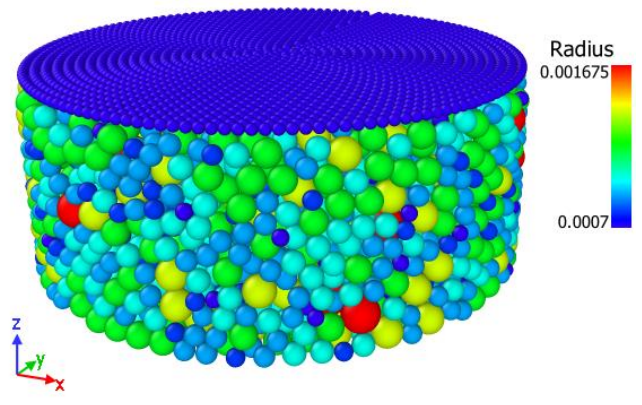

Fig. 8. DEM model of the oedometer test

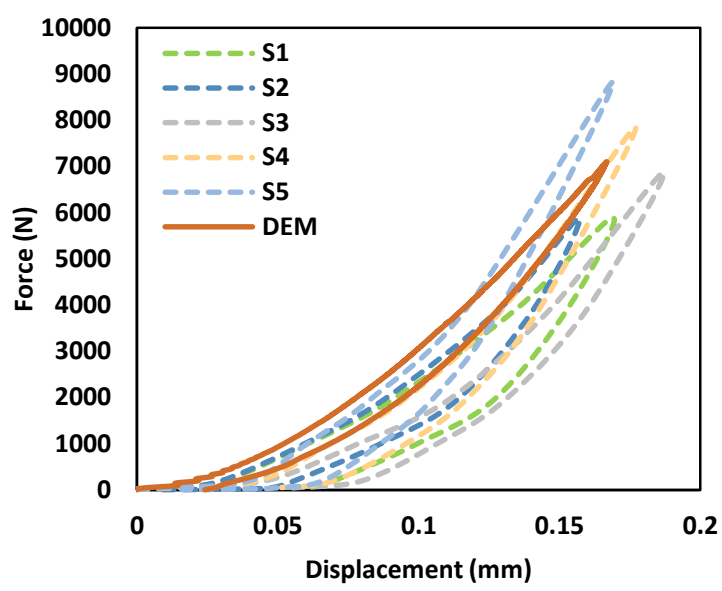

Fig. 9. Comparison of the test results between the experiments and DEM modelling

\section{Conclusions}

The present study represents an attempt to characterise the Filter Sand grade 5.5 by direct measurement approach to be used in the DEM modelling. The parameters essential for the modelling such as young's modulus, coefficient of restitution and friction angle are measured by doing appropriate tests. The parameters obtained from the direct measurement approach are used to determine the contact parameters for modelling the soil in the DEM.

The DEM model is created to represent the experimental setup and it was found that the force displacement results obtained by the DEM modelling is in good agreement with the experimental results. The effect of particle shape though not considered, did not affect the results partially because the experiment is onedimensional compression which does not lead to the failure of the specimen.

\section{References}

1. P.A. Cundall, O.D.L. Strack, Géotechnique, 29, 4765 (1979)

2. M. Marigo, E.H. Stitt, KONA Powder Part. J., 32, 236-252 (2015)

3. T.A.H. Simons, R. Weiler, S. Strege, S. Bensmann, M. Schilling, A. Kwade, Procedia Eng., 102, 741748 (2015)
4. D.M. Cole, J.F. Peters, Granul. Matter, 9, 309-321 (2007)

5. D.M. Cole, M.A. Hopkins, Granul. Matter, 19, 1-16 (2017)

6. H. Hertz, J. Für Die Reine Und Angew. Math., 156$171(1881)$

7. H. Mindlin R D, Deresiewicz, Timoshenko's Shear Coefficient for Flexural Vibrations of Beams (1953) doi:10.1002/nav.3800080206

8. A. Di Renzo, F.P. Di Maio, Chem. Eng. Sci., 59, 525-541 (2004)

9. B. Peng, B. Peng, Discrete Element Method (DEM) Contact Models Applied to Pavement Simulation, Virginia Polytechnic Institute and State University (2014)

10. N.P. Daphalapurkar, F. Wang, B. Fu, H. Lu, R. Komanduri, Exp. Mech., 51, 719-728 (2011)

11. B.J. Briscoe, L. Fiori, E. Pelillo, J. Phys. D Appl. Phys., 31, 2395-2405 (1988)

12. D. MA, Sci. China Ser. E., 47, 398 (2004)

13. Y.C. Chung, H.H. Liao, S.S. Hsiau, Powder Technol., 237, 53-66 (2013)

14. D. Wang, M. Servin, T. Berglund, K.O. Mickelsson, S. Rönnbäck, Powder Technol., 283, 475-487 (2015)

15. M. Ucgul, J.M. Fielke, C. Saunders, Biosyst. Eng., 121, 105-117 (2014)

16. M. Ucgul, J.M. Fielke, C. Saunders, Soil Tillage Res., 144, 220-227 (2014)

17. A. Haron, K.A. Ismail, Coefficient of restitution of sports balls: A normal drop test, IOP Conf. Ser. Mater. Sci. Eng., 36 (2012)

18. S. Plimpton, J. Comput. Phys., 117, 1-19 (1995) 